\title{
Hand Gesture Classification in Transradial Amputees Using the Myo Armband Classifier*
}

\author{
M. Cognolato ${ }^{1,2}$, M. Atzori ${ }^{1}$, D. Faccio ${ }^{3}$, C. Tiengo ${ }^{3}$, F. Bassetto ${ }^{3}$, R. Gassert ${ }^{2}$ and H. Müller ${ }^{1}$
}

\begin{abstract}
Dexterous hand prostheses controlled via surface electromyography represent the most advanced non invasive functional restorative solution for hand amputees. However, control difficulties, comfort problems and high costs are still the main limitations of such commercial devices. The high cost can represent a barrier that is difficult to overcome, especially for pediatric populations and in developing countries. Low-cost technology was successfully used in the hand prosthetics field in recent years. In previous work, a low-cost gesture recognition armband called Myo showed promising results for hand gesture classification tasks in intact subjects. Most of these applications were based on machine learning techniques applied to the Myo raw data. However, the classifier provided with the Myo is able to identify five hand gestures, providing capabilities as a myoelectric control system. No studies have quantitatively investigated its performance in subjects with hand amputation, yet. The aim of this study is to quantitatively evaluate the performance of the Myo hand gesture classifier in hand amputees. Three subjects with hand amputation were asked to attempt performing the five pre-set hand gestures. Each gesture was repeated three times with the arm in three different postures. The subjects did not perform any training and did not receive any feedback. Overall classification accuracy for the four hand gestures based on electromyographic data ranged between $50 \%$ and $97 \%$. A clear relation between the length of the residual limb and the classification accuracy was observed. The results show that the Myo built-in classifier can provide good performance when tested on hand amputees, supporting its applicability as a low-cost myoelectric control system.
\end{abstract}

\section{INTRODUCTION}

The loss of an upper limb can substantially reduce the quality of life of a person. It directly affects the physical capabilities of the subject but also plays an important role in social life and social activities. It was estimated that around 41,000 people were living with a major upper limb loss in the USA in 2005 [1]. Transradial amputation (namely an amputation between the wrist and elbow) has been reported to be the most common level of upper limb amputation in both the adult and pediatric population. Traumatic injury in adults and congenital limb loss in the pediatric population are reported as primary causes of upper limb loss [2]. Prosthetics

\footnotetext{
*This work was partially supported by the Swiss National Science Foundation Sinergia project \# 410160837 MeganePro.

${ }^{1}$ M. Cognolato, M. Atzori and $\mathrm{H}$. Müller are with the Information Systems Institute, University of Applied Sciences Western Switzerland (HES-SO Valais), Sierre, Switzerland. \{matteo.cognolato, manfredo.atzori, henning.mueller\}@hevs.ch

${ }^{2}$ M. Cognolato and R. Gassert are with the Rehabilitation Engineering Laboratory, Department of Health Sciences and Technology, ETH Zurich, Zurich, Switzerland. \{matteo.cognolato, roger.gassert\}@hest.ethz.ch

${ }^{3}$ D. Faccio, C. Tiengo and F. Bassetto are with the Clinic of Plastic Surgery, Padova University Hospital, Padova, Italy. \{diego. faccio, cesare.tiengo, franco.bassetto\}@unipd.it
}

companies and scientific research are advancing towards active hand prostheses that can act almost like real hands but there are still several limitations and challenges to be solved. Cosmetic, body-powered and myoelectric prostheses are the primary solutions for hand amputation. Cosmetic prostheses only have aesthetic function whereas body-powered and myoelectric prostheses are also functional. Body-powered prostheses are actuated by the subject's body while myoelectric prostheses are externally powered devices controlled by the electrical signals of the subject's remnant muscles measured with a technique called electromyography (EMG). The control of a commercial myoelectric prosthetic hand is usually performed by measuring the EMG signals from the skin (sEMG) of the residual limb with 2 electrodes. sEMG signals are used to open and close the prosthesis as well as to shift between the grasp types [3]. The control system of most commercial dexterous myoelectric prosthetic hands is sequential: the user selects the desired grasp among a predefined set (in most cases via co-contraction of agonist and antagonist muscles) and controls its opening and closing via myoelectric signals. Despite the remarkable advancements made in the last years, functionality (e.g. control difficulties) and comfort (e.g. weight, heat) are areas in which users felt the need for improvements [4], [5], [6]. Furthermore, commercial dexterous myoelectric hand prostheses are often very expensive (in the order of tens of thousands of dollars [7], [8]) and not systematically fully covered by public healthcare systems or reimbursement policies of insurance companies [9], [5].

Advancements in scientific research highlighted that the use of a larger number of sEMG electrodes placed around the residual limb together with machine learning techniques can increase the capabilities of the myoelectric control [3], [10], [11], [12]. However, these advancements are not yet the standard in commercial devices. To the best of our knowledge, only one myoelectric control system based on pattern recognition techniques is commercially available since 2013 [13], [3], [14].

In recent years low-cost technology such as 3D printing, electronic prototyping platforms and low-cost sensors were successfully applied to the field of hand prosthetics. In particular, a wireless low-cost gesture recognition armband (Myo, Thalmic Labs [15]) was released a few years ago. This device was tested on intact subjects for hand movement recognition tasks and in myoelectric control pipelines, showing good results [16], [17], [18], [19]. However, a quantitative evaluation of the device and its hand gesture classification algorithm on hand amputees is still missing. 
A comparison of six sEMG setups showed that the performance of the Myo armband on the classification of 41 hand gestures in intact subjects is comparable to the results that can be achieved with expensive and advanced sEMG sensors [16]. In particular, a difference of only $5 \%$ in classification accuracy was found using two Myo armbands for a protocol of 50 different gestures. The setup based on a single Myo showed a classification accuracy gap of $25 \%$ which, considering the number of hand gestures to be classified and the characteristics of the device, is still a notable result. Other groups have also successfully employed the Myo armband for intention detection. In Atasoy et al. raw sEMG data from the Myo and an Artificial Neural Network (ANN) were used to control a 24 degree of freedom (DoF) 3D printed hand prosthesis. The performance was evaluated on eighteen intact subjects, reaching about $80 \%$ accuracy on 7 grasps [18]. In Novak et al. an automatic approach to predict failures in dexterous myocontrol was proposed and tested with the Myo armband on an intact subject [17]. Mendez and colleagues compared the Myo armband performance in hand gesture classification on intact subjects, concluding that, despite the limited bandwidth, the Myo could be suitable for pattern recognition applications [20]. In Ryser et al. the Myo armband was successfully used for myoelectric intention detection in stroke survivors [21].

Regarding the use of the Myo by hand amputees, a thorough quantitative evaluation of its capabilities is still missing, even though some tests on prosthetic hands and virtual reality (VR) can be found in scientific publications and on the Internet [22], [23], [24]. The Myo itself is composed of an array of sEMG electrodes and designed to recognize five pre-set gestures. Therefore, it can be used as hand gesture myocontroller. The aim of this study is to quantitatively investigate the performance of the Myo gesture recognition armband with hand amputees. The results can lead to the development of affordable and simple-to-use controllers for subjects with hand amputation.

\section{METHODS}

The purpose of this study is to evaluate the potential of the Myo for recognizing hand gestures in hand amputees. Three subjects with hand amputation were recruited for this study. All the subjects were male with traumatic injury as cause of the amputation. Subjects 1 and 3 have had the disability for more than 15 years whereas the second subject underwent amputation in 2011. Subjects 1 and 2 had an amputation of the dominant hand. The remaining forearm percentage is calculated as the ratio between the stump length and the length of the intact forearm (Table I). Regarding prosthesis usage, subjects 2 and 3 use a bodypowered device, whereas subject 1 uses a non-polyarticulate (with one DoF) myoelectric hand prosthesis. All subjects reported to wear the prosthesis for the entire day and to have never used the Myo armband before. Subject characteristics are summarized in Table I.

\section{A. Experimental setup}

The acquisition setup is composed of the Myo armband and an acquisition laptop. The Myo is a wireless low-cost gesture recognition armband based on sEMG and inertial measurement unit (IMU) data (Fig. 1a). It is equipped with eight medical grade stainless steel dry sEMG electrodes and a nine-axis IMU. It weighs 93 grams and has an adjustable circumference ranging from 19 to 34 centimeters. The Myo armband is provided with a real-time hand gesture recognition algorithm. The Myo is capable to distinguish between 6 classes: 4 default hand gestures, a gesture called double tap and the resting posture. The default gestures are: fist (hand closed), wave in (wrist flexion), wave out (wrist extension), and fingers spread (hand open). The double tap (performed by tapping thumb to the forefingers twice in quick succession) is used to lock/unlock the device, enabling/disabling the identification of gestures (Fig. 1b). Specifications about the classifier e.g. the length of the time window are not provided by the manufacturer. The data are transmitted via Bluetooth from the Myo to the acquisition laptop and acquired with a custom made acquisition software written in $\mathrm{C}++$. Hand gesture data were acquired through the Myo software development kit (SDK) and timestamped using the high performance counter of the acquisition laptop. The use of the double tap gesture to lock/unlock the device was disabled and the hand gestures recognized by the Myo were continuously recorded. All the data were recorded at $20 \mathrm{~Hz}$ and stored in text files.

\section{B. Experimental protocol}

Before starting the acquisition each subject received a thorough written and oral explanation of the experiment and was asked to sign an informed consent form. The acquisition protocol was developed according to the principles expressed in the Declaration of Helsinki and it was approved by the Ethics Commission of the Province of Padova (Italy). Each subject was asked to wear the Myo armband on the residual limb (Fig. 1a) and, after the warm-up phase required by the device, a custom calibration was performed using the Myo armband manager software. During the calibration, a video of the hand gestures to be performed was shown to the subject who was asked to attempt to repeat the movement as naturally as possible with the missing hand. The calibration includes the four default hand gestures and the resting state. No calibration is provided for the double tap gesture.

The protocol consists of performing five hand gestures (the four default ones plus the double tap) with the arm in three postures: horizontal pointing in front of the subject, vertical along the body, and pointing up. Each hand gesture was repeated three times for each arm posture, resulting in nine repetitions per gesture. The sequence of movements was not randomized in order to let the subjects focus on performing the requested gesture and to limit wrong movement repetitions. The calibration was performed only once at the beginning of the test. During the data acquisition, vocal instructions about the hand gesture to be performed were given to the subject (referred to as gesture request in the text) 
TABLE I

CHARACTERISTICS OF THE SUBJECTS WITH HAND AMPUTATION RECRUITED FOR THE STUDY.

\begin{tabular}{|c||c||c||c||c||c||c||c||c||c|}
\hline & Gender & Age & $\begin{array}{c}\text { Height } \\
{[\mathrm{cm}]}\end{array}$ & $\begin{array}{c}\text { Weight } \\
{[\mathrm{Kg}]}\end{array}$ & Handedness & $\begin{array}{c}\text { Amputation } \\
\text { Side }\end{array}$ & $\begin{array}{c}\text { Year of } \\
\text { amputation }\end{array}$ & $\begin{array}{c}\text { Residual } \\
\text { forearm length }\end{array}$ & $\begin{array}{c}\text { Type of } \\
\text { prosthesis }\end{array}$ \\
\hline Sbj 1 & M & 48 & 190 & 99 & Right & Right & 1999 & $90 \%$ & Myoelectric \\
\hline Sbj 2 & M & 73 & 175 & 88 & Right & Right & 2011 & $52 \%$ & $\begin{array}{c}\text { Body- } \\
\text { Powered }\end{array}$ \\
\hline Sbj 3 & M & 36 & 170 & 65 & Right & Left & 2000 & $27 \%$ & $\begin{array}{c}\text { Body- } \\
\text { Powered }\end{array}$ \\
\hline
\end{tabular}

The information regarding the requested hand gesture was annotated in the acquisition software, leading to the ground truth signal. A number from one to five was assigned to each gesture, as shown in Fig. 1b. Fig. 2 shows an example of data acquired for a single arm posture. Since the purpose was to exploit the performance of the Myo gesture recognition system, no training phase was performed and no feedback was given to the subject during the acquisitions.

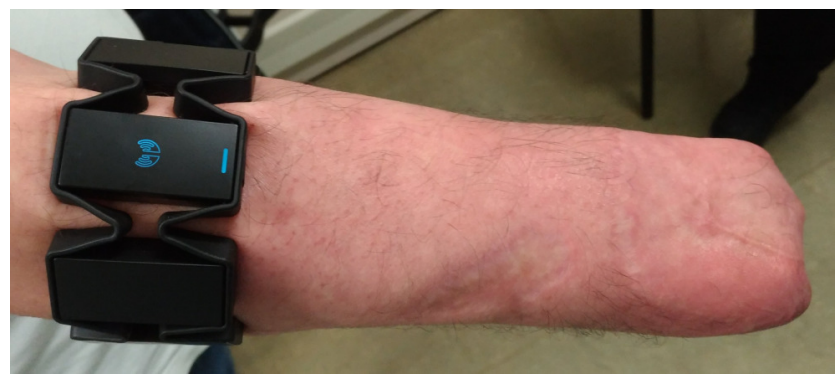

(a)

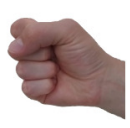

1. Fist

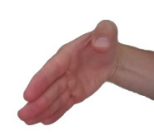

2. Wave

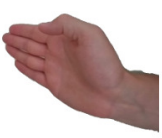

3. Wave Out

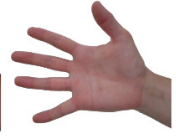

4. Fingers Spread
5. Double Tap

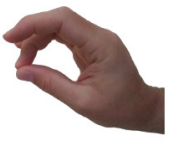

(b)

Fig. 1. (a) The Myo armband worn by subject 1. (b) The five gestures recognized by the Myo armband and acquired in this study.

\section{Data analysis}

The aim of this study is to investigate if the low-cost commercial gesture recognition Myo armband can be used as myocontrol system by subjects with hand amputation. Thus, the analysis focuses on the capabilities of the Myo classifier to recognize the hand gestures in hand amputees. Misclassifications and robustness of the recognition are also investigated. The analysis includes all the hand gesture repetitions, independently from the arm posture, leading to a total of 9 repetitions for each hand gesture. The double tap gesture is very particular: it cannot be calibrated and the movement has to be done in quick succession. Thus, not surprisingly, it was never classified correctly and we decided to not include it in the data analysis. As shown in Fig. 2, the
Myo returns the identified class (a hand gesture or the resting state) for each time sample, thus multiple hand gestures can be recognized within the same gesture request. An example is given in Fig. 2: gestures 3,1 and finally 4 were recognized during the first repetition, when the requested gesture was the fourth (fingers spread). In order to take into account this variability, the output of the classifier for each gesture request was restricted to the hand gesture that was recognized for the longest time. For instance, in the example mentioned before the gesture number 3 (wave out) was considered the output of the classifier because, among the three, it is the one that was recognized for the longest time.

\section{RESULTS}

The results show that the Myo armband classifier can provide reasonably good results on transradial amputees, depending on their clinical characteristics. In order to better represent the classification results, a confusion matrix was computed for each subject (Fig. 3a, 3b, and 3c). The gestures fist, wave in, wave out, and fingers spread were used as classes. The output was considered unclassified when only the resting state was identified during an entire gesture request. The overall classification accuracy is calculated as the proportion of hand gestures correctly classified with respect to the total number. The sensitivity (the true positive rate) and the precision (the positive predictive value) are evaluated for each gesture. As summarized in Table II,

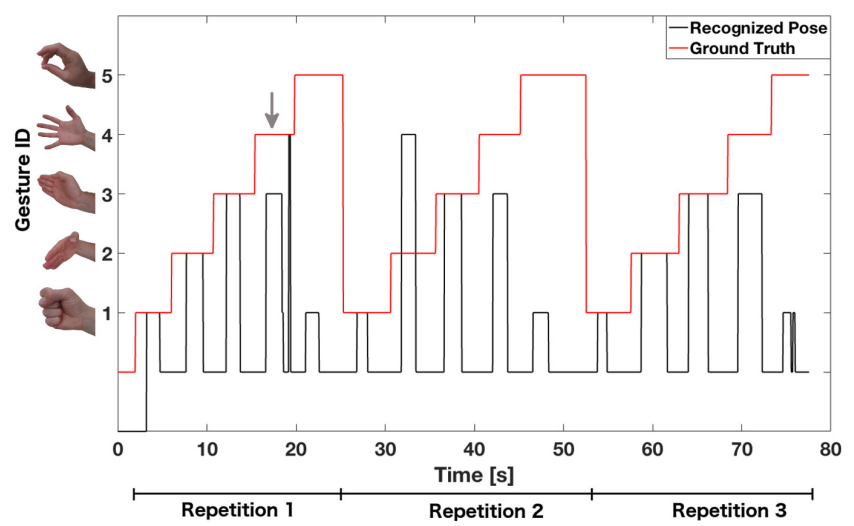

Fig. 2. Example of data acquired at a constant arm posture. The red line represents the ground truth. The black line represents the hand gesture as classified by the Myo. The arrow indicates the example given in Sec. II-C. 
subject 1, 2 and 3 respectively obtained overall classification accuracies of $97.2 \%, 66.7 \%$ and $50.0 \%$. Except for one case, the four default gestures were always correctly recognized for subject 1 , achieving an overall classification accuracy of $97.2 \%$. Considering specific hand gestures, the lowest sensitivity and precision was obtained by subject 3 for the fingers spread gesture. Only for subject 3 some repetitions were considered unclassified. This occurred five times for gesture 3 (wave out) and a single time for gesture 4 (fingers spread). Therefore, the sensitivity for these gestures was calculated considering the unclassified gestures as incorrect classification. The confusion matrices allow to investigate the performance of the classifier for each class. Only one misclassification occurred in subject 1: in one case gesture 2 (wave in) was classified as fist. In subject 2, the most problematic hand gesture was gesture 3 (wave out) which eight times out of nine was classified as fingers spread. Gesture 4 (fingers spread) was often misclassified in subject 3 as well. However, for this subject, gesture 4 (fingers spread) was classified as gesture 3 (wave out) most of the time, and gesture 3 remained mainly unclassified.

TABLE II

GESTURE CLASSIFICATION SENSITIVITY AND OVERALL ACCURACY.

\begin{tabular}{|c||c||c||c||c||c|}
\hline & Fist & $\begin{array}{c}\text { Wave } \\
\text { In }\end{array}$ & $\begin{array}{c}\text { Wave } \\
\text { Out }\end{array}$ & $\begin{array}{c}\text { Fingers } \\
\text { Spread }\end{array}$ & $\begin{array}{c}\text { Overall } \\
\text { Accuracy }\end{array}$ \\
\hline Sbj 1 & $100 \%$ & $88.9 \%$ & $100 \%$ & $100 \%$ & $97.2 \%$ \\
\hline Sbj 2 & $88.9 \%$ & $77.8 \%$ & $11.1 \%$ & $88.9 \%$ & $66.7 \%$ \\
\hline Sbj 3 & $100 \%$ & $66.7 \%$ & $33.3 \% *$ & $0.0 \% *$ & $50.0 \%$ \\
\hline
\end{tabular}

Classification accuracies quantify the performance of the recognition. However, another important factor to consider in this field is robustness, particularly for real-time classification. In order to evaluate the robustness of the recognition, the number of times several hand gestures were recognized within a single gesture request was investigated. As in the previous analyses, all repetitions of the four default hand gestures at any arm posture were included. Two times out of $36(5.55 \%)$ multiple hand gestures were recognized within a single instruction for subject 1 . Higher percentages were obtained for subjects 2 and 3: seven (19.44\%) and four $(11.11 \%)$ times, respectively. However, the high percentage of subject 2 is actually the result of repetitive classification of the correct hand gesture within the same gesture request (three occasions).

\section{DISCUSSION}

The purpose of this study is to investigate the use of the low-cost Myo armband as hand gesture recognition device for subjects with hand amputation. While the use of the Myo as pure hardware device to collect sEMG and IMU data was already evaluated on intact subjects, no quantitative investigations of the Myo classifier were found for hand amputees. The subjects did not perform any training (except

\footnotetext{
*Values computed taking into account the unclassified gestures as incorrect classification.
}

\begin{tabular}{|c|c|c|c|c|c|}
\hline 1. Fist & $\begin{array}{c}9 \\
25.0 \%\end{array}$ & $\begin{array}{c}1 \\
2.8 \%\end{array}$ & $\begin{array}{c}0 \\
0.0 \%\end{array}$ & $\begin{array}{c}0 \\
0.0 \%\end{array}$ & $\begin{array}{l}90.0 \% \\
10.0 \%\end{array}$ \\
\hline $\begin{array}{l}\text { 2. Wave } \\
\text { In }\end{array}$ & $\begin{array}{c}0 \\
0.0 \%\end{array}$ & $\begin{array}{c}\mathbf{8} \\
22.2 \%\end{array}$ & $\begin{array}{c}0 \\
0.0 \%\end{array}$ & $\begin{array}{c}0 \\
0.0 \%\end{array}$ & $\begin{array}{l}100 \% \\
0.0 \%\end{array}$ \\
\hline $\begin{array}{c}\text { 3. Wave } \\
\text { Out }\end{array}$ & $\begin{array}{c}0 \\
0.0 \%\end{array}$ & $\begin{array}{c}0 \\
0.0 \%\end{array}$ & $\begin{array}{c}\mathbf{9} \\
25.0 \%\end{array}$ & $\begin{array}{c}0 \\
0.0 \%\end{array}$ & $\begin{array}{l}100 \% \\
0.0 \%\end{array}$ \\
\hline $\begin{array}{l}\text { 4. Fingers } \\
\text { Spread }\end{array}$ & $\begin{array}{c}0 \\
0.0 \%\end{array}$ & $\begin{array}{c}\mathbf{0} \\
0.0 \%\end{array}$ & $\begin{array}{c}0 \\
0.0 \%\end{array}$ & $\begin{array}{c}9 \\
25.0 \%\end{array}$ & $\begin{array}{l}100 \% \\
0.0 \%\end{array}$ \\
\hline & $\begin{array}{l}100 \% \\
0.0 \%\end{array}$ & $\begin{array}{l}88.9 \% \\
11.1 \%\end{array}$ & $\begin{array}{l}100 \% \\
0.0 \%\end{array}$ & $\begin{array}{l}100 \% \\
0.0 \%\end{array}$ & $\begin{array}{c}97.2 \% \\
2.8 \%\end{array}$ \\
\hline
\end{tabular}

\begin{tabular}{|c|c|c|c|c|c|}
\hline \multicolumn{6}{|c|}{ (b) } \\
\hline \multirow[b]{2}{*}{ 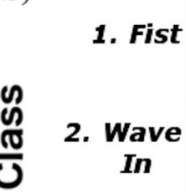 } & $\begin{array}{c}\mathbf{8} \\
22.2 \%\end{array}$ & $\begin{array}{c}0 \\
0.0 \%\end{array}$ & $\begin{array}{c}0 \\
0.0 \%\end{array}$ & $\begin{array}{c}0 \\
0.0 \%\end{array}$ & $\begin{array}{l}100 \% \\
0.0 \%\end{array}$ \\
\hline & $\begin{array}{c}1 \\
2.8 \%\end{array}$ & $\begin{array}{c}7 \\
19.4 \%\end{array}$ & $\begin{array}{c}0 \\
0.0 \%\end{array}$ & $\begin{array}{c}1 \\
2.8 \%\end{array}$ & $\begin{array}{l}77.8 \% \\
22.2 \%\end{array}$ \\
\hline $\begin{array}{c}\text { 3. Wave } \\
\text { Out }\end{array}$ & $\begin{array}{c}0 \\
0.0 \%\end{array}$ & $\begin{array}{c}1 \\
2.8 \%\end{array}$ & $\begin{array}{c}1 \\
2.8 \%\end{array}$ & $\begin{array}{c}0 \\
0.0 \%\end{array}$ & $\begin{array}{l}50.0 \% \\
50.0 \%\end{array}$ \\
\hline $\begin{array}{l}\text { 4. Fingers } \\
\text { Spread }\end{array}$ & $\begin{array}{c}0 \\
0.0 \%\end{array}$ & $\begin{array}{c}1 \\
2.8 \%\end{array}$ & $\begin{array}{c}8 \\
22.2 \%\end{array}$ & $\begin{array}{c}8 \\
22.2 \%\end{array}$ & $\begin{array}{l}47.1 \% \\
52.9 \%\end{array}$ \\
\hline & $\begin{array}{l}88.9 \% \\
11.1 \%\end{array}$ & $\begin{array}{l}77.8 \% \\
22.2 \%\end{array}$ & $\begin{array}{l}11.1 \% \\
88.9 \%\end{array}$ & $\begin{array}{l}88.9 \% \\
11.1 \%\end{array}$ & $\begin{array}{l}66.7 \% \\
33.3 \%\end{array}$ \\
\hline
\end{tabular}

\begin{tabular}{|c|c|c|c|c|c|}
\hline 1. Fist & $\begin{array}{c}\mathbf{9} \\
25.0 \%\end{array}$ & $\begin{array}{c}\mathbf{0} \\
0.0 \%\end{array}$ & $\begin{array}{c}0 \\
0.0 \%\end{array}$ & $\begin{array}{c}2 \\
5.6 \%\end{array}$ & $\begin{array}{l}81.8 \% \\
18.2 \%\end{array}$ \\
\hline $\begin{array}{c}\text { 2. Wave } \\
\text { In }\end{array}$ & $\begin{array}{c}0 \\
0.0 \%\end{array}$ & $\begin{array}{c}6 \\
16.7 \%\end{array}$ & $\begin{array}{c}0 \\
0.0 \%\end{array}$ & $\begin{array}{c}0 \\
0.0 \%\end{array}$ & $\begin{array}{l}100 \% \\
0.0 \%\end{array}$ \\
\hline $\begin{array}{c}\text { 3. Wave } \\
\text { Out }\end{array}$ & $\begin{array}{c}0 \\
0.0 \%\end{array}$ & $\begin{array}{c}1 \\
2.8 \%\end{array}$ & $\begin{array}{c}3 \\
8.3 \%\end{array}$ & $\begin{array}{c}6 \\
16.7 \%\end{array}$ & $\begin{array}{l}30.0 \% \\
70.0 \%\end{array}$ \\
\hline \multirow[t]{3}{*}{$\begin{array}{l}\text { 4. Fingers } \\
\text { Spread }\end{array}$} & $\begin{array}{c}0 \\
0.0 \%\end{array}$ & $\begin{array}{c}2 \\
5.6 \%\end{array}$ & $\begin{array}{c}1 \\
2.8 \%\end{array}$ & $\begin{array}{c}0 \\
0.0 \%\end{array}$ & $\begin{array}{l}0.0 \% \\
100 \%\end{array}$ \\
\hline & $\begin{array}{l}100 \% \\
0.0 \%\end{array}$ & $\begin{array}{l}66.7 \% \\
33.3 \%\end{array}$ & $\begin{array}{l}33.3 \% \\
66.7 \%\end{array}$ & $\begin{array}{c}0.0 \%^{*} \\
100 \%\end{array}$ & $\begin{array}{l}50.0 \% \\
50.0 \%\end{array}$ \\
\hline & Fist & $\begin{array}{c}\text { Wave } \\
\text { In }\end{array}$ & $\begin{array}{c}\text { Wave } \\
\text { Out }\end{array}$ & $\begin{array}{l}\text { Fingers } \\
\text { Spread }\end{array}$ & \\
\hline
\end{tabular}

Target Class

Fig. 3. Confusion matrices for subject 1 (a), 2 (b) and 3 (c). The number of hand gestures and the percentage with respect to the total number of repetitions (36) are reported in each cell. The columns show the correct class and the rows correspond to the predicted class. The diagonal shows the correct estimations of hand gestures while misclassifications are reported in the off-diagonal. The last column shows precision and false discovery rate for each predicted class and the last row reports sensitivity and false negative rate for each correct class. The overall accuracy is reported in the bottom right cell. 
for the calibration) and they were not provided with any kind of feedback on their performance. Therefore, the obtained results reflect the baseline performance of the system. The results show that the four default gestures are recognizable by the Myo classifier also in subjects with hand amputation. No subject was able to perform the double tap gesture. This can be explained by the fact that this gesture uses orientation and rotation data from the IMU to identify when it is triggered whereas the 4 default gestures use pure-EMG data. Therefore, the required pattern to trigger the double tap is hardly reproducible by a hand amputee. The results suggest that the four default hand gestures of the Myo armband can be classified also in subjects with hand amputation, even though clinical parameters (such as remaining forearm percentage) can substantially influence the results.

The overall hand gesture recognition accuracies range from $97.2 \%$ to $50.00 \%$. Subject 1 was able to perform the four pre-set default hand gestures almost perfectly without any training nor feedback. This subject was the only one using a myoelectric hand prosthesis on a regular basis and this factor might have positively influenced the performance. The daily use of a myoelectric hand prosthesis keeps the subject trained on using the remnant muscles of the residual limb [25]. On the other hand, good results were obtained also for subject 2 who uses a body-powered device. The lowest classification accuracy was obtained for subject 3 . At a first glance, this could seem a poor result. However, this subject does not use a commercial myoelectric hand prosthesis mainly due to difficulties of identifying the optimum control site locations on the residual limb. Therefore, achieving $100 \%$ classification accuracy for the fist gesture can be considered a very promising result. Control site locations from which reliable sEMG signals can be recorded are required to control most of the commercial myolectric hand prostheses. The myocontrol of such commercial devices often relies on two sEMG electrodes placed on antagonist muscles groups (wrist flexors and extensors are common ones). The contraction of these muscles is used to open and close the prosthetic hand, regardless the selected grasp. Commonly, an amputee can control the closing of the prosthetic hand by contracting the wrist flexor muscles whereas the opening is controlled by the contraction of the wrist extensors. Therefore, the myocontrol generally implemented in commercial myoelectric hand prostheses is substantially different from the one used in this study with the Myo. The Myo allows to implement an approach similar to the one used in scientific research: the application of pattern recognition techniques to myoelectric signals recorded from an array of electrodes placed around the residual limb allows the direct identification of the gesture that the subject aims to perform.

While comparing the obtained results with the length of the residual limb, a clear relation was observed: the longer the residual limb, the higher the classification accuracy. The same result was found also in Atzori et al., evaluating the relationship between clinical parameters and hand gesture classification accuracy in a sample of eleven hand amputees [26], using an advanced laboratory sEMG setup.
Regarding the robustness of the classification, the results show that in some cases multiple hand gestures were recognized by the Myo classifier within a single gesture request. This is reasonable considering that these acquisitions were performed with no training nor feedback. Both can potentially improve accuracy and robustness of the classification. In a closed-loop user-machine interaction, the user learns how to perform the command in order to be correctly recognized by the system [27]. A phase of training could be particularly beneficial for those subjects not regularly using a myoelectric prosthesis. The training could help them to improve their ability to control the remnant muscles in the residual limb, generating distinguishable and reliable EMG activations [25].

The results show that the built-in Myo classifier provides good performance also in subjects with hand amputation, supporting its applicability as an inexpensive myocontrol system and opening new possible scenarios of applications. For example, these results show that the Myo armband can be used as is to myoelectrically control a hand prosthesis with an on-off control strategy (in which the actuators are either turned on or off [28]). This application can actually improve the intuitiveness of the control for opening/closing the prosthetic hand: by associating the closing of the prosthesis to the fist gesture and the opening to the finger spread gesture the amputee would actually close/open the prosthesis while attempting to close/open the missing hand. Furthermore, a proportional myoelectric control system for a hand prosthesis could be built on top of the Myo classifier. The hand gesture classification could be performed by the built-in Myo classifier and the sEMG intensity could be used to proportionally control a prosthesis.

The aforementioned applications could also be implemented in a virtual reality (VR) and augmented reality (AR) environment, to create for instance a prosthetic VR or AR training tool. Functional training and an early fitting of the prosthesis are crucial factors that can increase prosthesis acceptance [29]. It has also been shown that the use of visual feedback given in a VR or AR environment can enhance hand prosthesis rehabilitation and training [30], [31]. However, to the best of our knowledge, no VR or AR applications for hand prosthetic training are currently publicly available nor used in the clinical setting. Therefore, the use of a low-cost wearable gesture recognition device, such as the Myo armband, can support the development of affordable and portable training applications, e.g. VR applications for smartphones, providing hand amputees with a training tool for myoelectric control.

\section{CONCLUSIONS}

The built-in Myo hand gesture classifier showed good performance on subjects with hand amputation, making it a good candidate for creating a low-cost and almost ready to use myoelectric control system for externally powered prosthetic hands. Since the hand gestures recognized by the Myo can be obtained in real-time by using the Myo SDK or the Bluetooth protocol, it can be particularly suitable 
for $3 \mathrm{D}$ printed hand prostheses as well as for affordable and portable myolectric control training VR/AR environments. For instance, an open-source electronic prototyping platform can be used to control robotic devices such as $3 \mathrm{D}$ printed hand prostheses or orthoses based on the hand gesture recognized by the Myo. Most of the 3D printed hand prostheses have limited mechanical and control capabilities compared to commercial devices. Nevertheless, such devices can restore basic but essential hand functionalities, which could substantially improve the quality of life of subjects with amputation or congenital deficiency of the hand. Pattern recognition systems started to be released recently and they represent an opportunity for the future of amputees, but they are still being improved and they are usually expensive and complex. The good performance shown by the Myo armband classifier on hand amputees can be particularly useful in prosthetics research and applications where costs are critical (i.e. small research groups, studies or usage by pediatric patients or in developing countries), since in these cases the Myo armband allows developing effective and simple-to-use myoelectric control systems for prosthetic devices.

\section{ACKNOWLEDGMENT}

The authors would like to thank the subjects participating in this study as well as the Swiss National Science Foundation for partially supporting this work via the Sinergia project \# 410160837 MeganePro.

\section{REFERENCES}

[1] K. Ziegler-Graham, E. J. MacKenzie, P. L. Ephraim, T. G. Travison, and R. Brookmeyer, "Estimating the Prevalence of Limb Loss in the United States: 2005 to 2050," Archives of Physical Medicine and Rehabilitation, vol. 89, no. 3, pp. 422-429, 2008.

[2] D. J. Atkins, D. C. Heard, and W. H. Donovan, "Epidemiologic Overview of Individuals with Upper-Limb Loss and Their Reported Research Priorities," JPO Journal of Prosthetics and Orthotics, vol. 8, no. 1, pp. 2-11, 1996.

[3] M. Atzori and H. Müller, "Control Capabilities of Myoelectric Robotic Prostheses by Hand Amputees: A Scientific Research and Market Overview." Frontiers in systems neuroscience, vol. 9, p. 162, 2015.

[4] S. Ritchie, S. Wiggins, and A. Sanford, "Perceptions of cosmesis and function in adults with upper limb prostheses: a systematic literature review." Prosthetics and orthotics international, vol. 35, no. 4, pp. 332-41, 2011.

[5] F. Cordella, A. L. Ciancio, R. Sacchetti, A. Davalli, A. G. Cutti, E. Guglielmelli, and L. Zollo, "Literature Review on Needs of Upper Limb Prosthesis Users," Frontiers in Neuroscience, vol. 10, p. 209, may 2016.

[6] E. Biddiss, D. Beaton, and T. Chau, "Consumer design priorities for upper limb prosthetics," Disabil. Rehabil. Assist. Technol., vol. 2, no. 6, pp. 346-357, 2007.

[7] D. K. Blough, S. Hubbard, L. V. McFarland, D. G. Smith, J. M. Gambel, and G. E. Reiber, "Prosthetic cost projections for servicemembers with major limb loss from Vietnam and OIF/OEF." Journal of rehabilitation research and development, vol. 47, no. 4, pp. 387402, 2010.

[8] D. Van Der Riet, R. Stopforth, G. Bright, and O. Diegel, "An overview and comparison of upper limb prosthetics," IEEE AFRICON Conference, 2013.

[9] E. Biddiss, P. McKeever, S. Lindsay, and T. Chau, "Implications of prosthesis funding structures on the use of prostheses: experiences of individuals with upper limb absence," Prosthetics and Orthotics International, vol. 35, no. 2, pp. 215-224, jun 2011.

[10] S. Micera, J. Carpaneto, and S. Raspopovic, "Control of hand prosthesis using peripheral information," Biomedical Engineering, IEEE Reviews in, vol. 3, pp. 48-68, 2010.
[11] M. Atzori, A. Gijsberts, C. Castellini, B. Caputo, A.-G. M. Hager, S. Elsig, G. Giatsidis, F. Bassetto, and H. Müller, "Electromyography data for non-invasive naturally-controlled robotic hand prostheses." Scientific data, vol. 1, p. 140053, 2014.

[12] D. Farina, N. Jiang, H. Rehbaum, A. Holobar, B. Graimann, H. Dietl, and O. C. Aszmann, "The extraction of neural information from the surface EMG for the control of upper-limb prostheses: Emerging avenues and challenges," IEEE Transactions on Neural Systems and Rehabilitation Engineering, vol. 22, no. 4, pp. 797-809, 2014.

[13] "Coapt, LLC." [Online]. Available: https://www.coaptengineering.com/

[14] D. Farina and S. Amsüss, "Reflections on the present and future of upper limb prostheses," Expert Review of Medical Devices, vol. 13, no. 4 , pp. 321-324, 2016.

[15] "Myo, Thalmic Labs Inc." [Online]. Available: https://www.myo.com/

[16] S. Pizzolato, L. Tagliapietra, M. Cognolato, M. Reggiani, H. Müller, and M. Atzori, "Comparison of six electromyography acquisition setups on hand movement classification tasks," PLOS ONE, vol. 12, no. 10, pp. 1-16, 2017.

[17] M. Nowak, S. Engel, and C. Castellini, "A preliminary study towards automatic detection of failures in myocontrol," in MEC17 - A Sense of What's to Come A, no. August, 2017, p. ID \# 82.

[18] A. Atasoy, E. Kaya, E. Toptas, S. Kuchimov, E. Kaplanoglu, and M. Ozkan, "24 DOF EMG Controlled Hybrid Actuated Prosthetic Hand," pp. 5059-5062, 2016.

[19] S. Masson, F. Fortuna, F. Moura, and D. Soriano, "Integrating Myo armband for the control of myoelectric upper limb prosthesis," $X X V$ Congresso Brasileiro de Engenharia Biomédica, no. October, 2016.

[20] I. Mendez, B. W. Hansen, C. M. Grabow, E. J. Smedegaard, N. B. Skogberg, X. J. Uth, A. Bruhn, B. Geng, and E. N. Kamavuako, "Evaluation of the Myo armband for the classification of hand motions," IEEE International Conference on Rehabilitation Robotics, pp. 12111214, 2017.

[21] F. Ryser, T. Bützer, J. P. Held, O. Lambercy, and R. Gassert, "Fully embedded myoelectric control for a wearable robotic hand orthosis," IEEE International Conference on Rehabilitation Robotics, pp. 615621, 2017.

[22] "Virtual Reality Prosthetics." [Online]. Available: https://vrprosthetics.shu.ac.uk/

[23] "Meet the man with a myo controlled robotic arm, Myo Blog." [Online]. Available: http://developerblog.myo.com/meet-theman-with-a-myo-controlled-robotic-arm/

[24] "DLR VITA Project." [Online]. Available: $\quad$ http://www.dlr.de/rm/en/desktopdefault.aspx/tabid12023/\#gallery/29007

[25] C. Cipriani, C. Antfolk, M. Controzzi, G. Lundborg, B. Rosen, M. C. Carrozza, and F. Sebelius, "Online myoelectric control of a dexterous hand prosthesis by transradial amputees," IEEE Transactions on Neural Systems and Rehabilitation Engineering, vol. 19, no. 3, pp. 260-270, 2011.

[26] M. Atzori, A. Gijsberts, C. Castellini, B. Caputo, A.-G. M. Hager, S. Elsig, G. Giatsidis, F. Bassetto, and H. Müller, "Effect of clinical parameters on the control of myoelectric robotic prosthetic hands," Journal of Rehabilitation Research and Development, vol. 53, no. 3, pp. 345-358, 2016.

[27] E. A. Corbett, K. P. Kording, and E. J. Perreault, "Real-time evaluation of a noninvasive neuroprosthetic interface for control of reach," IEEE Transactions on Neural Systems and Rehabilitation Engineering, vol. 21, no. 4, pp. 674-683, 2013.

[28] A. Fougner, O. Stavdahl, P. J. Kyberd, Y. G. Losier, and P. A. Parker, "Control of upper limb prostheses: Terminology and proportional myoelectric controla review," IEEE Trans. Neural Syst. Rehabil. Eng., vol. 20, no. 5, pp. 663-677, 2012.

[29] L. Resnik, M. R. Meucci, S. Lieberman-Klinger, C. Fantini, D. L. Kelty, R. Disla, and N. Sasson, "Advanced upper limb prosthetic devices: Implications for upper limb prosthetic rehabilitation," Archives of Physical Medicine and Rehabilitation, vol. 93, no. 4, pp. 710-717, 2012.

[30] T. Wada and T. Takeuchi, "A Training System for EMG Prosthetic Hand in Virtual Environment," Proceedings of Human Factors and Ergonomics Society 52nd Annual Meeting, vol. 52, no. 27, pp. 21122116, 2008.

[31] F. Anderson and W. F. Bischof, "Augmented reality improves myoelectric prosthesis training," International Journal on Disability and Human Development, vol. 13, no. 3, pp. 349-354, 2014. 